\title{
Effects of a Novel Differentiation Factor on the Development of Catecholamine Traits in Noncatecholamine Neurons from Various Regions of the Rat Brain: Studies in Tissue Culture
}

\author{
Lorraine lacovitti \\ Center for Neurological Research of the Department of Neurology, Hahnemann University School of Medicine, \\ Philadelphia, Pennsylvania 19102
}

\begin{abstract}
The muscle-derived differentiation factor called MDF initiated expression of the catecholamine (CA) enzyme tyrosine hydroxylase (TH) in non-CA neurons isolated from a variety of regions in the rat brain. Specifically, subpopulations of neurons from the striatum, collicular plate, and cerebellum were TH-immunoreactive after an overnight exposure to MDF in culture. The number of immunopositive cells was greatest in the striatum, where more than half of all plated neurons expressed the enzyme. In contrast, MDF had no effect on the central neurons of the hippocampus or on peripheral sensory neurons. In ${ }^{3} \mathrm{H}$-thymidine studies, only brain neurons that had already withdrawn from mitosis expressed TH. These cells remained open to the epigenetic influence of MDF only during a brief and defined critical period that appears to be timed intrinsically. Without daily replenishment of MDF, expression of the enzyme disappeared after several days in culture, suggesting that MDF was rapidly depleted or degraded in vitro. However, in the continued presence of MDF, TH expression was maintained indefinitely, thus producing a permanent alteration in phenotype. Moreover, a single exposure to MDF during the critical period was sufficient to render neurons permanently receptive to the molecule so that $\mathrm{TH}$ expression could be reinitiated many days later. It is postulated that a memory of this biochemical interaction was established in these neurons, making transmitter phenotypic plasticity possible at later stages.
\end{abstract}

During the developmental process known as differentiation, neurons acquire the cell-specific traits that together constitute their phenotype. One of the critical phenotypic decisions neurons make is the selection of which neurotransmitters they will use at thcir synapses. Regrettably, our understanding of the molecular steps that lead to this decision remains inadequate. However, over a decade of work on the PNS has provided valuable insight into certain aspects of the process. In these studies on rat sympathetic neurons, environmentally derived substances have been shown to play a key role in directing the final choice of neurotransmitter (Bunge et al., 1978; Le Douarin, 1980; Yamamori et al., 1989; Schotzinger and Landis, 1990).

\footnotetext{
Received Aug. 24, 1990; revised Feb. 25, 1991; accepted Mar. 11, 1991.

This work was supported by NIH Grant 1RO1 NS2404-04 and a Ben Franklin Foundation Award. Special thanks to L. Lyandvert and T. Simuni for excellent technical assistance.

Correspondence should be addressed to Dr. lacovitti, Department of Neurology, Hahnemann University, Broad \& Vine, M.S. 423, Yhiladelphia, PA 19102.

Copyright (C) 1991 Society for Neuroscience $0270-6474 / 91 / 112403-07 \$ 03.00 / 0$
}

Thus, sympathetic neurons, which normally express only catecholamine-associated traits, also display cholinergic phenotypic characters when grown in the presence of certain agents (Johnson et al., 1976; Patterson and Chun, 1977; Iacovitti et al., 1981; Kessler, 1985). Conversely, cholinergic neurons of the parasympathetic nervous system express adrenergic traits if exposed to the appropriate environmental cues in vitro (Iacovitti et al., 1985; Teitelman et al., 1985) and in vivo (Coulombe and Bronner-Fraser et al., 1986; Landis et al., 1987). These agents have been called differentiation factors because of their ability to influence phenotypic decisions without affecting cell survival.

To date, several putative differentiation factors with activities in the PNS have been described (Sah and Matsumoto, 1987; Adler et al., 1989; Yamamori et al., 1989). Because of their extraordinarily low concentration in tissues, isolation and characterization of the pure molecules have been extremely difficult. Recently, however, the cholinergic neuronal differentiation factor has been purified to homogeneity (Weber, 1981; Fukada, 1985 ) and a cDNA encoding it has been sequenced (Yamamori et al., 1989). As described previously, this protein, which is secreted by cultured rat heart cells, promotes expression of cholinergic traits in sympathetic neurons (Patterson and Chun, 1977). Interestingly, in addition to neurons, this molecule also influences the differentiation of myeloid and other cell types, suggesting that some differentiation factors may play multiple roles in development.

While it has long been anticipated that such factors also regulate the differentiation of brain neurons, a candidate substance has only recently been proposed. This putative molecule, called muscle-derived differentiation factor or MDF, has profound effects on the expression of transmitter-specific genes in cultured brain neurons (Iacovitti et al., 1989). As its name implies, MDF has been extracted and partially purified from rat muscle cells (L6 cell line). In culture, it has the unique capacity to initiate transcription of the gene for tyrosine hydroxylase $(\mathrm{TH})$, the first and limiting enzyme in catecholamine (CA) biosynthesis, in cells where the gene is normally quiescent. Without change in neuronal survival, MDF increases by 10 -fold the levels of mRNAcncoding $\mathrm{TH}$ and its corresponding protein in cultured cells from the embryonic rat cerebral cortex (lacovitti et al., 1989). While many known substances can amplify the levels of $\mathrm{TH}$ protein in CA neurons (for review, see Zigmond et al., 1989), this is the first example of a factor that initiates expression of a CA gene in non-CA neurons from brain.

As is the case for many known growth factors (Perez-Polo, 1987), MDF exerts its influence on the genome only at a very precise stage in the development of the neuron. Thus, the ce- 
rebral cortex of rats at $13 \mathrm{~d}$ gestation are dramatically affected by MDF; however, by $16 \mathrm{~d}$ gestation, cortical neurons are no longer responsive to the molecule (Iacovitti et al., 1987). The existence of a critical period for the actions of MDF raises questions about the regulation of this timed event and the role that it plays in influencing the initial choice of neurotransmitter and the potential for transmitter plasticity at later stages.

This study sought to answer some of the fundamental questions raised by our earlier studies on the biological actions of MDF, including whether other neurons from the CNS as well as the PNS respond to MDF and, if so, whether the response is universally the same. Experiments were designed to investigate the timing of the critical period for the effects of MDF and the regulatory site for this event. Finally, the long-term consequences of MDF treatment on the phenotypic fate of neurons were studied. The findings of the present study demonstrate that the effects of MDF are confined to the CNS, and there, only to specific subpopulations of neurons. As in the cortex, neurons from elsewhere in the brain are responsive to MDF only during a critical period that appears to be intrinsically timed in each cell type. As well as promoting the initial appearance of $\mathrm{TH}$, MDF perpetuated expression of the enzyme when it was continually present in the culture. This resulted in a permanent alteration in neurotransmitter phenotype. Moreover, a single exposure to MDF during the operative period renders neurons MDF-responsive at later times. It is suggested that this early developmental "experience" establishes a biochemical memory of induction in the cell and thereby provides a mechanism for phenotypic plasticity at later stages in life.

\section{Materials and Methods}

Tissue culture. Pregnant Sprague-Dawley rats were purchased from Taconic Lab Animals at $12 \mathrm{~d}$ gestation $\pm 12 \mathrm{hr}$ fertilization day $=\mathrm{em}$ bryonic day (E) 0 . On E13, E14, E16, or E18, rats were anesthetized with pentobarbital, and the embryos were removed. After dissection of the brain from the cranium, the cerebral cortices, striatum, collicular plate, hippocampus, and cerebellum were isolated. After removal of the meninges, tissue was incubated in $\mathrm{Ca}^{2+}, \mathrm{Mg}^{2+}$-free Hanks' balanced salt solution (CMF-HBSS) for $10 \mathrm{~min}$ at $37^{\circ} \mathrm{C}$ in a clinical rotator $(40 \mathrm{rpm})$. The incubation mixture was replaced with a $0.01 \%$ trypsin solution (in CMF-HBSS) and incubated for another $15 \mathrm{~min}$, rinsed twice in Leibovitz medium (L-15), and placed in culture medium containing Dulbecco's minimum essential medium, 10\% fetal calf serum (Irvine Scientific), glucose $(6 \mathrm{mg} / \mathrm{ml})$, glutamine $(204 \mu \mathrm{g} / \mathrm{ml})$, and penicillin/ streptomycin $(100 \mathrm{U} / \mathrm{ml})$. Peripheral dorsal root ganglia (DRG) isolated from embryos between E16 and E19 were stripped of adherent connective tissue and incubated in CMF-IIBSS for $10 \mathrm{~min}$ at $37^{\circ} \mathrm{C}$ before being transferred to a $0.25 \%$ trypsin solution for $30 \mathrm{~min}$. Tissue was rinsed in LI5 and then placed in media as described above with the addition of $100 \mathrm{ng} / \mathrm{ml}$ purified nerve growth factor (kind gift of Dr. E. M. Johnson, Washington University Medical School, St. Louis, MO). Cells were dissociated by trituration through a reduced-bore glass pipette and plated onto glass Lab Tek culture dishes coated either with polymerized collagen or with polyornithine (precoated for $24 \mathrm{hr}$ at room temperature at a concentration of $0.1 \mathrm{mg} / \mathrm{ml}$ in $15 \mathrm{~mm}$ borate buffer, pH 8.4). The cellular plating density was approximately $5 \times 10^{5}$ cells/ dish. Striatal cells formed fewer cell aggregates on polyornithine than on collagen; thus, in radioautography experiments, which required that all cells be evenly coated with emulsion, cells were grown on polyornithine. In all other experimental manipulations, collagen was used.

Preparation of partially purified $M D F$. To prepare soluble extract of muscle, cultures of nearly confluent L6 muscle cells were grown for 10 $\mathrm{d}$ in vitro, harvested, homogenized in $0.01 \mathrm{M}$ sodium phosphate buffer (pH 7.2,60 $\times 10^{6}$ cells $/ \mathrm{ml}$ ) for $1-2$ min with a polytron, and centrifuged at $40,000 \times g$ for $1 \mathrm{hr}$ to separate soluble and insoluble fractions. The clear supernatants were collected, the protein levels were determined, and the supernatants were treated with acid. By lowering the $\mathrm{pH}$ to 2 with $\mathrm{HCl}$ for 1 min on ice and then raising it back to $\mathrm{pH} 6$ with $\mathrm{NaOH}$,
$90 \%$ of the protein was precipitated with no loss of MDF activity. This fraction was then passed over a reverse-phase $\mathrm{C} 18$ column and MDF was recovered in the flow through fraction. This step removed a yellow chromophore (probably carnitive, which is concentrated in muscle) that is toxic to the cells in culture. This fraction was then passed over a DEAE-cellulose anion exchange column (Whatman DE-52). MDF was weakly bound under these conditions, and $60 \%$ of the activity could be recovered after elution from the column. This $\approx 10,000$-fold-purified molecule(s) was concentrated by lyophilization with $90 \%$ recovery and resuspended in culture media ( $\mathrm{pH} 7.8$ ) for use in experiments.

Immunocytochemistry. Cultures were fixed in $4 \%$ paraformaldehyde in $0.1 \mathrm{M}$ phosphate buffer $(\mathrm{pH} \mathrm{7.4)}$ and processed with antibodies to TH (1:5000 dilution, kind gift of Dr. T. H. Joh, Cornell University Medical College, New York, NY) using the immunoperoxidase/avidinbiotin complex (ABC) method of staining (Elite Vectakit). Except where stated otherwise, $\mathrm{TH}^{+}$cell numbers were determined by counting stained cells in all microscopic fields on the culture dish. This was accomplished with the aid of an cycpicce rcticulc uscd at a $10 \times$ magnification.

Thymidine labeling. To determine whether cells in culture synthesize DNA, brain cells were plated as described, and after $1 \mathrm{hr}$ media containing $0.05 \mu \mathrm{Ci} / \mathrm{ml}$ of ${ }^{3} \mathrm{H}$-thymidine (specific activity, $78.0 \mathrm{Ci} / \mathrm{mmol}$ ) was added. After $1 \mathrm{~d}$ in vitro, cultures were fixed with $4 \%$ paraformaldehyde and processed for the immunohistochemical visualization of $\mathrm{TH}$ as well as the autoradiographic localization of incorporated thymidine (presence of silver grains over the nucleus). This double-labeling technique has been described previously (Teitelman et al., 1985).

\section{Results}

Effects of $M D F$ on $T H$ expression in various regions of the nervous system. Our earlier studies demonstrated that, in culture, the soluble muscle-derived factor, designated MDF, induces synthesis of TH and its encoding mRNA in 10\% of cultured neurons from the embryonic rat cerebral cortex (Iacovitti et al., 1989). The present study sought to determine whether MDF affected other non-C $\Lambda$ neurons of central and also peripheral origin. In these experiments, cultures of dissociated cells were established from various regions of the brain and PNS (see Materials and Methods). All cultures were stabilized on a complete media overnight and subsequently transferred into either control media or media supplemented with partially purified MDF. After $2 \mathrm{~d}$ in vitro, cultures were fixed and processed for the localization of TH antibodies.

As was previously observed in the cerebral cortex, cultures generated from other non-CA regions of the CNS, including striatum, collicular plate, and cerebellum, contained low percentages of TH-immunoreactive neurons even when grown in control media (Table 1). However, the number of TH neurons in these cultures was markedly increased by growing the cells in media containing MDF. The greatest increment was found in the striatum (52-fold) (Table 1), with approximately $50 \%$ of the neurons expressing $\mathrm{TH}$ after an overnight exposure to MDF (Fig. 1). Smaller increases were observed in the colliculus (fourfold) and cerebellum (fivefold) (Table 1). Interestingly, however, not all brain regions were MDF-responsive; cultures of hippocampal neurons were devoid of TH immunoreactive neurons under control conditions, and they did not express $\mathrm{TH}$ after MDF supplementation at any of the ages tested (Table 1).

In contrast to the dramatic actions of MDF on CNS neurons, no measurable effect was observed in sensory neurons of the PNS. Consequently, the number of TH-immunopositive neurons of the dorsal root ganglia was not increased by MDF treatment (Table 1).

These results suggest that specific populations of neurons from some but not all brain regions respond to MDF with enhanced TH expression, as evidenced by the unexpected appearance of TH immunoreactivity in cells. In contrast, non-CA sensory neu- 

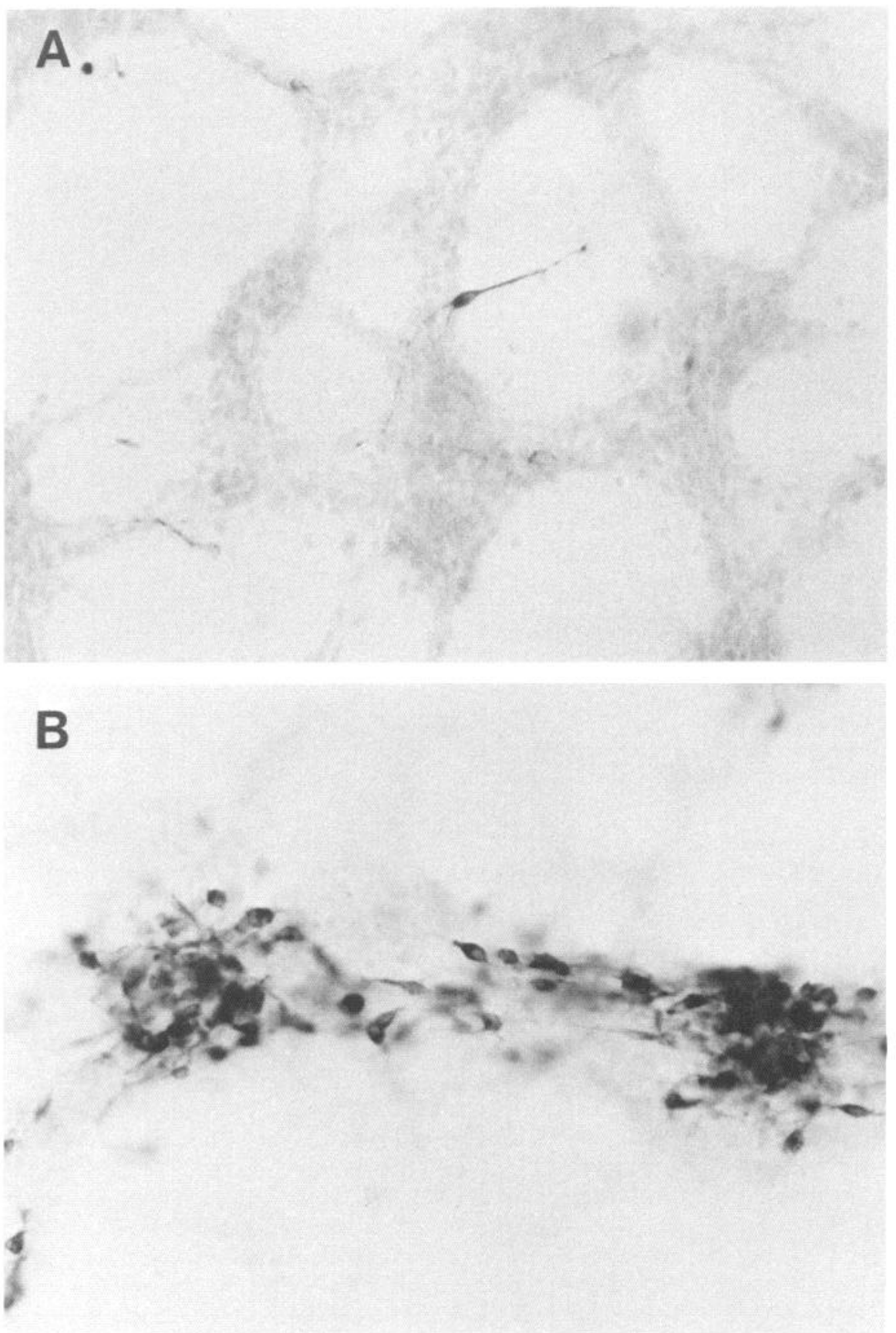

Figure 1. Effect of MDF on TH expression in cultures of E14 caudate nucleus. Photomicrographs are shown of striatal neurons grown either on control media $(A)$ or in the presence of MDF $(B)$ for $1 \mathrm{~d}$ before being processed for the immunocytochemical localization of $T H$. rons of the PNS do not express TH in response to MDF treatment in culture.

Effects of MDF on the critical period. Our earlier studies demonstrated that cerebral cortical neurons had the ability to respond to cues in culture and express TH only during a brief critical period in their embryogenesis (Iacovitti et al., 1987). To assess whether other MDF-responsive neurons shared this property of stage dependence, cultures were generated from a variety of brain regions isolated from embryos ranging in age from E14 to E20. As was found previously, neurons from other brain regions were MDF-competent only during restricted periods in their development (Fig. 2). This period occurred between E14 and E16 in collicular plate and cerebellar cultures. However, in striatal cultures, the critical period was protracted, extending at least up to the time of birth. The number of MDF-responsive neurons, however, steadily declined during this period.

For each brain region, the critical period appeared to correspond to the time just following peak withdrawal of neurons from the mitotic cycle (DeLong and Sidman, 1962; Altman, 1963; Berry et al., 1964; Fentress et al., 1981). This notion was tested by incubating neurons of varying age in media containing ${ }^{3} \mathrm{H}$-thymidine. After an 18-hr exposure, cultures were fixed and processed for simultaneous localization of TH by immunocytochemistry and ${ }^{3} \mathrm{H}$-thymidine incorporation by radioautography. Although the majority of unstained cells were labeled autoradiographically, no TH-immunoreactive neurons from any regions tested contained silver grains over their nuclei (data shown only for striatal neurons in Fig. 3). The absence of label 
Table 1. Comparison of MDF-induced changes in TH expression in neurons from the CNS and PNS

\begin{tabular}{llcl} 
& $\begin{array}{l}\text { MDF } \\
\text { treatment }\end{array}$ & $\begin{array}{c}\text { Number of TH } \\
\text { neurons/culture }\end{array}$ & $\begin{array}{l}\text { Fold } \\
\text { induction }\end{array}$ \\
\hline CNS & & & \\
Cerebral cortex & - & $1222 \pm 118$ & 12 \\
Striatum & + & $14,631 \pm 991$ & \\
& - & $431 \pm 78$ & 52 \\
Collicular plate & + & $22,542 \pm 769$ & \\
& + & $461 \pm 93$ & 4 \\
Cerebellum & + & $1,909 \pm 440$ & \\
& - & $129 \pm 28$ & 5 \\
Hippocampus & + & $658 \pm 104$ & \\
& - & 0 & 0 \\
PNS & + & 0 & \\
Dorsal root ganglion & - & 2 & 0
\end{tabular}

Embryonic rats were dissected at varying developmental ages. Brain cells from cerebral cortex, striatum, cerebellum, collicular plate, and hippocampus (E13-20) and peripheral neurons from dorsal root ganglia (E16-19) were dissociated and plated in culturc. All cultures were stabilized on a complete media overnight and subsequently transferred into either control media or media supplemented with partially purified MDF. After incubation for $2 \mathrm{~d}$ in vitro, cultures were fixed and processed for the localization of TH antibodies and the number of TH-immunoreactive neurons was counted in all microscopic fields. Values represent means \pm SEM of triplicate cultures from two separate platings.

was not due to the dilution of ${ }^{3} \mathrm{H}$ that occurs after successive divisions since the length of the cell cycle is $11 \mathrm{hr}$ (von Waechter and Jaensch, 1973). Instead, the absence of radioisotope incorporation indicates that these cells have not synthesized DNA and thus probably are not replicating while in culture. The cxtended critical period observed in striatum presumably reflects the fact that striatal neurons continue to be generated, though to a lesser extent, even into the first week of postnatal life (Fentress et al., 1981).

Regulation of the timing of the critical period. The fact that neurons remain responsive to MDF for only a brief critical period after withdrawing from mitosis raises the question as to whether the timing of this event is intrinsically preprogrammed in the cell or occurs as the result of some critical interaction with other developing cells. To distinguish between these possibilities, cortical cells from E13 rat were placed in culture, thus isolating them from cellular interactions in vivo. Neurons were allowed to "age," presumably beyond the critical period, by growing them in culture for $7 \mathrm{~d}$ on control media. Cultures were

\section{Table 2. Timing of critical period for MDF induction of TH is set} by internal clock

\begin{tabular}{llc}
$\begin{array}{l}\text { Age } \\
\text { of } \\
\text { donor } \\
\text { cortex }\end{array}$ & $\begin{array}{l}\text { Days in vitro } \\
\text { prior to } \\
\text { MDF treatment }\end{array}$ & $\begin{array}{l}\text { Number of } \\
\mathrm{TH}^{+} \\
\text {neurons/culture }\end{array}$ \\
\hline E13 & 1 & $38,946 \pm 4662$ \\
E13 & 7 & $11 \pm 3$
\end{tabular}

The cerebral cortex was isolated from rats at $13 \mathrm{~d}$ of gestation, dissociated, and grown in culture on control media. After 1 or $7 \mathrm{~d}$ in culture, media was replaced with MDF-supplemented feed. One day later, cultures were fixed and stained with TH antibodies, and the number of TH neurons/culture was determined. Values represent means \pm SEM of duplicate cultures from two separate platings.

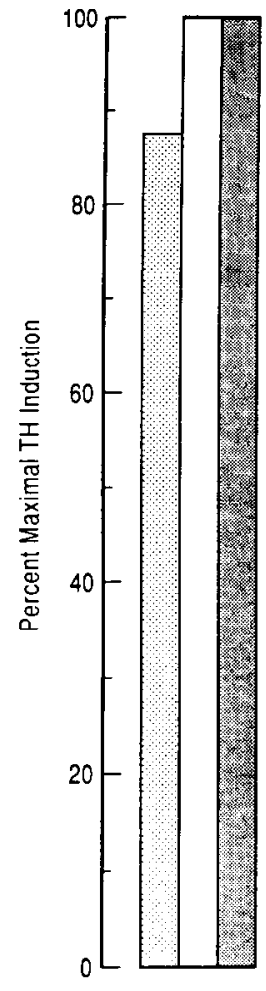

14

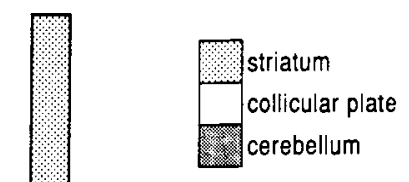

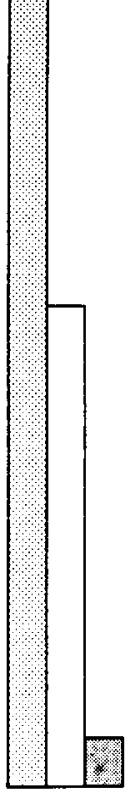

16

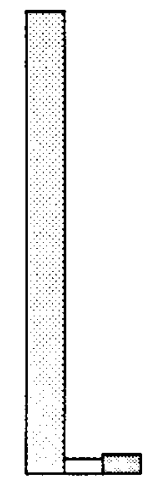

18

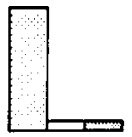

20
Age of Donor Embryo

(days gestation)

Figure 2. Cultures of dissociated striatum, collicular plate, and cerebellum were established from embryos at a variety of different developmental stages. After $1 \mathrm{~d}$ in vitro, cultures were supplemented overnight with MDF before $T H$ immunocytochemical processing. Values are expressed as a percentage of maximal induction (number of $\mathrm{TH}^{+}$neurons) for each brain region. Maximal values are provided in Table 1 .

then supplemented with MDF from culture day 7 to 9 before immunocytochemical analysis. Cultures treated in this manner were devoid of TH-positive neurons (Table 2), suggesting that the timing of the critical period may be internally regulated.

Effects of MDF on the fate of TH expression. Even when responsive neurons are exposed to MDF during their critical period, the induced expression of $\mathrm{TH}$ is short-lived, disappearing after only a few days in vitro (Iacovitti et al., 1987). To test whether MDF, in addition to initiating expression of TH, might also prolong the presence of the enzyme there, cerebral cortical cells were plated in culture either on media containing MDF or on control media. After $2 \mathrm{~d}$, several cultures from each group were fixed and processed for immunocytochemistry. All remaining cultures were maintained on control media until day 8 when they were fixed and stained, and the number of $\mathrm{TH}$ neurons was determined. As expected, after $2 \mathrm{~d}$ in vitro, MDFtreated cultures contained eightfold more $\mathrm{TH}$ neurons than did control cultures (Fig. 4). However, expression of the enzyme in MDF-supplemented cultures, as in controls, rapidly declined. By $7 \mathrm{~d}$ in vitro, only an occasional TH-positive neuron was present. To determine whether the loss of TH expression resulted from the depletion and/or degradation of MDF with increasing incubation time, some cultures of cortical cells were replenished daily with MDF-supplemented media. In these cultures, TH expression was maintained at $7 \mathrm{~d}$ in vitro (Fig. 4) and 


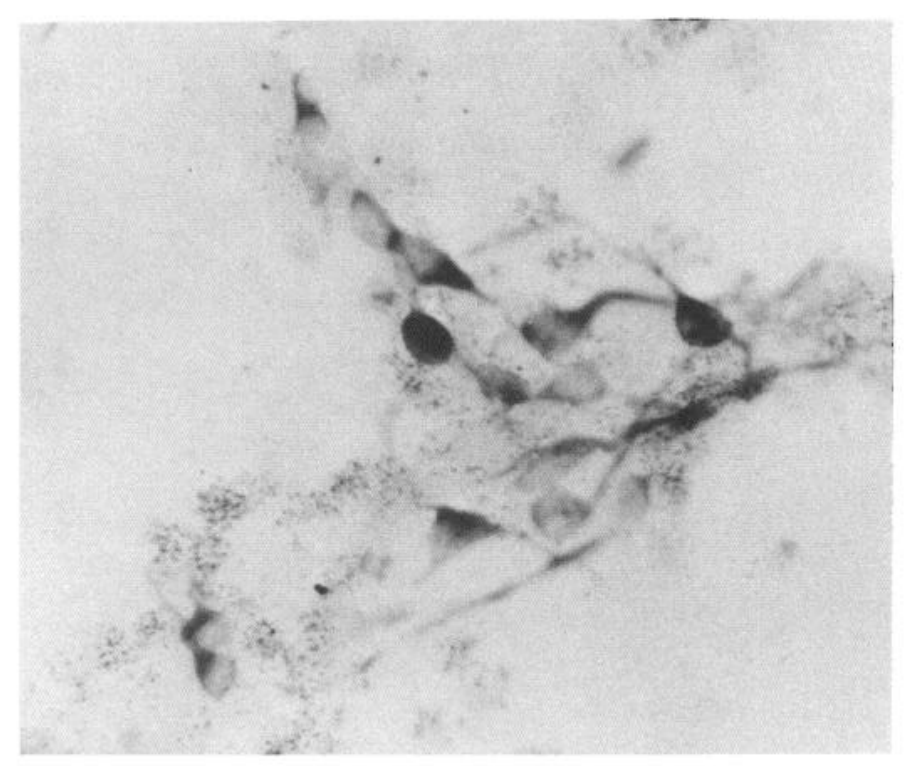

Figure 3. Combined radioautography of ${ }^{3} \mathrm{H}$-thymidine and $\mathrm{TH}$ immunocytochemistry in cultures of E14 striatum. Note that cells containing TH immunoreactivity do not contain silver grains over their nuclei.

at later times (14 d in culture; data not shown). These results suggest that MDF can both initiate and perpetuate TH expression indefinitely in cultured cortex but that the factor is rapidly depleted or degraded with time in vitro.

Finally, in this series of experiments, the possibility was examined that a single exposure to MDF during the critical period was sufficient to produce long-lasting effects on TH. To test this, cultures of E13 cortical cells were grown in MDF-containing media during the $2 \mathrm{~d}$ immediately following plating and then replaced with control media for the next $10 \mathrm{~d}$ before receiving a final overnight exposure to MDF. Cultures were then processed for immunocytochemical analysis. As demonstrated in Table 3, untreated neurons or neurons treated after close of the critical period were not affected by MDF. However, a single exposure to MDF during the critical period rendered cortical neurons permanently receptive, such that $\mathrm{TH}$ expression could be reinduced by MDF as much as $14 \mathrm{~d}$ later.

\section{Discussion}

It has previously been reported that the muscle-derived substance called MDF promotes the differentiation of CA traits in cultured neurons from the rat cerebral cortex (Iacovitti et al., 1989). The present study demonstrates that MDF produces a

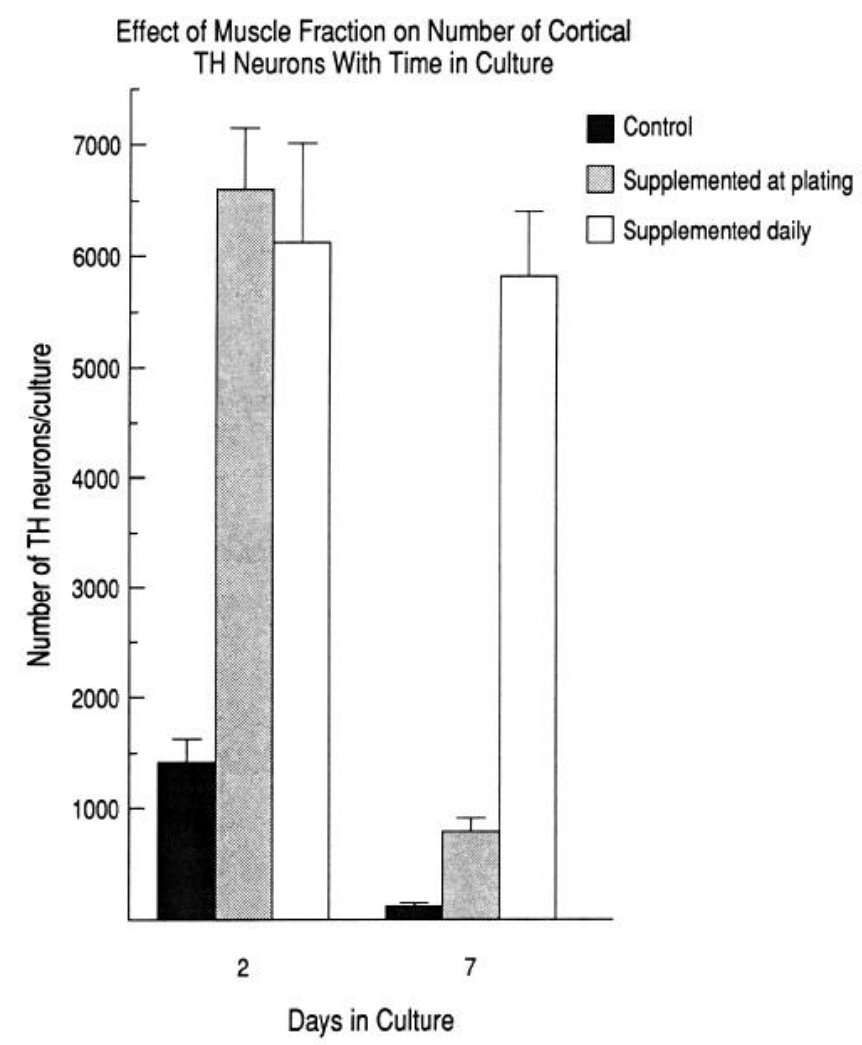

Figure 4. Cultures of dissociated E13 rat cerebral cortical cells were established in control media or media supplemented with MDF. After the initial treatment with MDF, supplemented cultures were split into two groups: one was fed daily with control media, the other with MDFsupplemented media. Cultures were fixed after 2 or $7 \mathrm{~d}$ in vitro, and then the number of TH-immunoreactive neurons was determined. Numbers represent means \pm SEM of triplicate cultures from two separate platings.

similar response in neurons from a wide variety of neural regions. Assessed in this study were areas from the CNS and PNS composed, nearly in their entirety, of neurons synthesizing neurotransmitters other than CAs. After overnight incubation with MDF, subsets of neurons from most but not all brain regions tested expressed $\mathrm{TH}$, the first enzyme in the CA biosynthetic pathway. The proportion of responsive cells varied from one region to another, with the most dramatic effect occurring in cultured striatal neurons. These responses were limited to neurons of the CNS; MDF had no observable effect on peripheral neurons from the sensory ganglia.

\begin{tabular}{|c|c|c|c|c|c|}
\hline \multirow{2}{*}{$\begin{array}{l}\text { Culture } \\
\text { number }\end{array}$} & \multicolumn{4}{|c|}{ MDF treatment (d) } & \multirow{2}{*}{$\begin{array}{l}\text { Number of } \mathrm{TH}^{+} \\
\text {neurons/culture }\end{array}$} \\
\hline & 0 & 1 & $2-12$ & 13 & \\
\hline 1 & - & - & - & - & $96 \pm 12$ \\
\hline 2 & - & + & + & + & $8649 \pm 752$ \\
\hline 3 & - & + & - & - & $111 \pm 29$ \\
\hline 4 & - & - & - & + & $116 \pm 16$ \\
\hline 5 & - & + & - & + & $6468 \pm 412$ \\
\hline
\end{tabular}

Cultures of E13 cerebral cortex were supplemented with MDF-containing media at various times in culture. After 14 $\mathrm{d}$, cultures were fixed and stained, and the number of $\mathrm{TH}^{+}$neurons was determined. Values represent means $\pm \mathrm{SEM}$ of duplicate cultures from two platings. 
As in the cerebral cortex (Iacovitti et al., 1987), other nonCA neurons were responsive to MDF only during well-delineated stages in their embryonic development. In all regions, this period corresponded to the time just following peak cell proliferation (DeLong and Sidman, 1962; Altman, 1963; Berry et al., 1964; Fentress et al., 1981). Thus, only neurons that had recently withdrawn from the mitotic cycle were susceptible to the effects of MDF in culture; neurons isolated from rats several days later in embryogenesis did not respond. This finding raised questions as to whether the timing of this event was genetically preprogrammed in the neuron or occurred as the result of some critical interaction with other developing cells. Indeed, during the early postmitotic period, many important cell-cell interactions are established in vivo, including contact with target and innervating neural structures. It is possible that a critical exchange with one or more of these cells fixed the phenotype of the neuron in vivo and, in so doing, precluded its ability to respond to MDF in culture. Arguing against this interpretation was the finding that neurons plated soon after their terminal mitosis and aged several days in culture before MDF treatment did not respond to treatment. Thus, similar to their in vivo counterparts, cultured cortical neurons developed a critical period for MDF without benefit of external influences. The findings of this study are, therefore, more consistent with the hypothesis that neurons contain an internal clock that regulates the timing of their responsiveness to MDF. However, because of the complexity of cell types in our cultures, we cannot eliminate the possibility that the critical period is dependent upon cellular interactions that are not removed by the cell culture process.

Even when competent neurons were treated with MDF during the critical period, TH expression was short-lived, disappearing after several days in culture. However, if replenished daily, MDF perpetuated the presence of the enzyme indefinitely, suggesting that (1) MDF was either rapidly depleted or degraded in vitro; (2) MDF was capable not only of initiating expression of TH but also of maintaining it; and (3) MDF could, in this way, effect a permanent change in neuronal phenotype.

Quite remarkably, a single exposure to the factor at the appropriate developmental stage allowed cortical neurons to reexpress TH after a second addition of MDF as much as $14 \mathrm{~d}$ later. As expected, neurons that did not receive this initial treatment remained unaffected by a later MDF challenge. These findings suggest that a "biochemical memory" of the effects of MDF was established in MDF-primed neurons. Priming of the neurons rendered them permanently receptive to the actions of the molecule and thus provided a mechanism for reexpression of the enzyme at later stages of life. It is possible that early interactions of the genome with differentiation factors are requisite steps for achieving phenotypic plasticity in the adult.

The findings of this study have addressed the issues of where in the nervous system MDF might be acting, when the molecule is most apt to be effective, and, in part, how MDF achieves its results. Less clear from these studies is why MDF so profoundly impacts certain neurons and not others. Do MDF-responsive neurons from these diverse locales in the brain share any one feature in common? One possibility under consideration is that responding neurons belong to a particular neurotransmitter class. Although direct proof is lacking, there is mounting circumstantial evidence to implicate the neurotransmitter GABA. Most compelling is the fact that over $90 \%$ of intrinsic neurons in the striatum are GABAergic in the adult rat (Mugnaini and Oertel, 1985). This fact, taken together with the finding in culture that more than half of the nondividing ncurons of the striatum express TH after MDF treatment, strongly suggests that TH-containing striatal neurons are (or will be) GABAergic. Further suggestive of a possible relationship between $\mathrm{TH}$ and GABA in these neurons are the results of preliminary double-labeling experiments on the cerebral cortex (L. Iacovitti, unpublished data). In that study, it was shown that $5 \mathrm{~d}$ after MDF treatment, when the proportion of $\mathrm{TH}$-positive neurons was declining and that of GABA-positive neurons was rising, occasional cells colabeled for both antigens. This suggested that $\mathrm{TH}$ expression was being downregulated in the same cortical neurons in which GABA was appearing. Experiments are currently in progress to substantiate these results further and to determine whether the coexpression of TH and GABA can be extended if cultures are maintained on MDF-supplemented media.

Interestingly, all regions that responded to MDF with an incrcase in the number of TH neurons always contained some TH-positive cells even under control culture conditions (in the absence of the factor). Conceivably, the appearance of $\mathrm{TH}$ in control cells might be due to the liberation of MDF or other differentiation factors during the dissociation procedure. Alternatively, this population may represent those occasional TH cells previously noted in vivo. Indeed, TH-immunoreactive neurons have been observed in all MDF-responsive brain regions, including the cerebral cortex (Gaspar et al., 1987; Kosaka et al., 1987a), striatum (Berger et al., 1985; Kosaka et al., 1987b; Satoh and Suzuki, 1990), and inferior colliculus (Jaeger and Joh, 1983). At least in the case of the cerebral cortex (Kosaka et al., 1987a), these cells also express GABA (Kosaka et al., 1987b). It is conceivable that these TH/GABA cells are vestiges of an earlier developmental stage. According to this hypothesis, certain neurons in the brain are capable of expressing genes related to both the CA and GABA transmittcr systems if exposed to appropriate differentiation signals at the correct times in development. In most cases, TH expression is downregulated, possibly as a result of the disappearance of CA-inducing factors, while GABA expression is upregulated. However, in some instances, coexpression is maintained even in the adult brain (Kosaka et al., 1987b). Proof of the existence of cells with this shared lineage awaits further investigation using double-labeling techniques.

\section{References}

Adler JE, Schleifer LS, Black IB (1989) Partial purification and characterization of a membrane-derived factor regulating neurotransmitter phenotypic expression. Proc Natl Acad Sci USA 86:1080-1083.

Altman J (1963) Autoradiographic investigation of all proliferation in the brains of rats and cats. Anat Rec 145:573-591.

Berger B, Verney C, Gaspar P, Febvret A (1985) Transient expression of tyrosine hydroxylase immunoreactivity in some neurons of the rat neocortex during postnatal development. Dev Brain Res 23:141-144.

Berry M, Rogers AW, Eayrs JT (1964) Pattern of cell migration during cortical histogenesis. Nature 203:591-593.

Bunge RP, Johnson M, Ross CD (1978) Nature and nurture in the development of the autonomic neurons. Science 199:1409-1415.

Coulombe JN, Bronner-Fraser M (1986) Catecholaminergic properties of cholinergic neurons acquire adrenergic neurotransmitters when transplanted into the embryo. Nature 324:569-572.

DeLong GR, Sidman RL (1962) Effects of eye removal at birth on histogenesis of the mouse superior colliculus: an autoradiographic with tritiated thymidine. J Comp Neurol 118:205-224.

Fentress JC, Stanfield BB, Cowan WM (1981) Observations on the development of the striatum in mice and rats. Anat Embryol 163: 275 298.

Fukada K (1985) Purification and partial characterization of a cholinergic neuronal differentiation factor. Proc Natl Acad Sci USA 82: 8795-8799. 
Gaspar P, Berger B, Febvret A, Vigny A, Krieger-Poulet M, Bori-Voltatorni C (1987) Tyrosine hydroxylase immunoreactive neurons in the human cerebral cortex: a novel catecholaminergic group? Neurosci Lett 80:257-262.

Iacovitti L, Joh TH, Park IH, Bunge RP (1981) Dual expressions of neurotransmitter synthesis in cultured autonomic neurons. J Neurosci $1: 685-690$.

Iacovitti L, Joh TH, Albert VR, Park DH, Reis DJ, Teitelman G (1985) Partial expression of catecholaminergic traits in cholinergic chick ciliary ganglia: studies in vivo and in vitro. Dev Biol 110:402-412.

Iacovitti L, Lee J, Joh TH, Reis DJ (1987) Expression of tyrosine hydroxylase in neurons of cultured cerebral cortex: evidence for phenotypic plasticity in neurons of the CNS. J Neurosci 7:1264-1270.

Iacovitti L, Evinger MJ, Joh TH, Reis DJ (1989) A muscle-derived factor(s) induces expression of a catecholamine phenotype in neurons of cultured rat cerebral cortex. J Neurosci 9:3529-3537.

Jaeger CB, Joh TH (1983) Transient expression of tyrosine hydroxylase in some neurons of the developing inferior colliculus of the rat. Dev Brain Res 11:128-132.

Johnson M, Ross D, Meyers M, Rees R, Bunge E, Wakshull E, Burton H (1976) Synaptic vesicle cytochemistry changes when cultured sympathetic neurons develop cholinergic interactions. Nature 262: 308-310.

Kessler JA (1985) Differential regulation of peptide and catecholamine characters in cultured sympathetic neurons. Neuroscience 15:827839.

Kosaka, T, Hama K, Nagatsu I (1987a) Tyrosine hydroxylase-immunoreactive intrinsic neurons in the rat cerebral cortex. Exp Brain Res 68:393-405.

Kosaka T, Kosaka K, Hataguchi Y, Nagatsu I, Wu J-Y, Ottersen OP, Storm-Mathisen J, Hama K (1987b) Catecholamine neurons containing GABA-like and/or glutamic acid decarboxylase-like immunoreactives in various brain regions of the rat. Exp Brain Res 66:191210.

Landis SC, Jackson PC, Freidieu JR, Thibault J (1987) Catecholaminergic properties of cholinergic neurons and synapses in adult rat ciliary ganglion. J Neurosci 7:3574-3587.
Le Douarin NM (1980) The neural crest. London: Cambridge IJP.

Mugnaini E, Oertel WH (1985) An atlas of the distribution of GABAergic neurons and terminals in the rat $\mathrm{CNS}$ as revealed by GAD immunohistochemistry. In: Handbook of chemical neuroanatomy, Vol 4, GABA and neuropeptides in the CNS, Pt I (Bjorklund A, Hokfelt T, eds). Amsterdam: Elsevier.

Patterson PH, Chun LLY (1977) The induction of acetylcholine synthesis in primary cultures of dissociated rat sympathetic neurons. I. Effects of conditioned medium. Dev Biol 56:263-280.

Perez-Polo JK (1987) Neuronal factors. Boca Raton, FL: CRC.

Sah DWY, Matsumoto SG (1987) Evidence for serotonin synthesis, uptake, and release in dissociated rat sympathetic neurons in culture. J Neurosci 7:391-399.

Satoh J, Suzuki K (1990) Tyrosine hydroxylase-immunoreactive neurons in the mouse cerebral cortex during the postnatal period. Dev Brain Res 53:1-5.

Schotzinger RJ, Landis SC (1990) Acquisition of cholinergic and peptidergic properties by sympathetic innervation of rat sweat glands requires interaction with normal target. Neuron 5:91-100.

Teitelman G, Joh TH, Grayson L, Park DH, Iacovitti L (1985) Cholinergic neurons of the chick ciliary ganglia express adrenergic traits in vivo and in vitro. $\mathrm{J}$ Neurosci 5:29-39.

von Waechter R, Jaensch B (1973) Generation times of the matrix cells during embryonic brain development: an autoradiographic study in rats. Brain Res 57:361-371.

Weber MJ (1981) A diffusable factor responsible for the determination of cholinergic functions in cultured sympathetic neurons. J Biol Chem 256:3447-3453.

Yamamori T, Fukada K, Aebersold R, Korsching S, Fann MJ, Patterson PH (1989) The cholinergic neuronal differentiation factor from heart cells is identical to leukemia inhibitory factor. Science 246:14121416.

Zigmond RE, Schwarzschild MA, Rittenhouse AR (1989) Acute regulation of tyrosine hydroxylase by nerve activity and by neurotransmitters via phosphorylation. Annu Rev Neurosci 12:415-461. 